\title{
Involvement of non-B cell-derived immunoglobulin $G$ in the metastasis and prognosis of salivary adenoid cystic carcinoma
}

\author{
JING PENG $^{1^{*}}$, HAI-CHENG WANG ${ }^{2 *}$, YANG LIU $^{3}$, JIU-HUI JIANG $^{4}$, \\ WAN-QI LV ${ }^{1}$, YUE YANG ${ }^{5}$, CUI-YING LI ${ }^{1}$ and XIAO-YAN QIU ${ }^{3}$ \\ ${ }^{1}$ Central Laboratory, Peking University School and Hospital of Stomatology, Beijing 100081; ${ }^{2}$ Department of \\ Oral Pathology, School and Hospital of Stomatology, Tongji University, Shanghai Engineering Research Center of \\ Tooth Restoration and Regeneration, Shanghai 200072; ${ }^{3}$ Department of Immunology, Key Laboratory of \\ Medical Immunology, Ministry of Health, School of Basic Medical Sciences, Peking University, Beijing 100191; \\ ${ }^{4}$ Department of Orthodontics, Peking University School and Hospital of Stomatology, Beijing 100081; \\ ${ }^{5}$ Department of Dentistry, Capital Medical University Xuanwu Hospital, Beijing 100053, P.R. China
}

Received March 9, 2016; Accepted February 27, 2017

DOI: $10.3892 / 01.2017 .6782$

\begin{abstract}
Cancer cell-derived immunoglobulin G (cancer-IgG) has been implicated in the pathogenesis and progression of various types of cancer. However, its role in salivary adenoid cystic carcinoma (SACC) remains unclear. The present study aimed to investigate the effects of cancer-IgG on metastasis and prognosis in 96 patients with SACC. Immunohistochemical staining showed that cancer- $\operatorname{Ig} G$ expression was present in all 96 individual SACC tissues. Additionally, high cancer-IgG expression was significantly correlated with metastasis, nerve invasion and recurrence in SACC $(\mathrm{P}<0.05)$. Moreover, cancer-IgG expression was significantly correlated with the survival duration of patients with SACC $(\mathrm{P}<0.05)$. Proliferation, cell motility and invasion all decreased significantly following knockdown of cancer-IgG in SACC cells $(\mathrm{P}<0.05)$ through population-doubling time, wound healing and transwell invasion assays. Additionally, cancer-IgG-knockdown in SACC cells induced the increased expression of E-cadherin and matrix metalloproteinase 9, and promoted the epithelial-mesenchymal transition, but decreased
\end{abstract}

Correspondence to: Professor Xiao-Yan Qiu, Department of Immunology, Key Laboratory of Medical Immunology, Ministry of Health, School of Basic Medical Sciences, Peking University, 38 Xueyuan Road, Beijing 100191, P.R. China

E-mail: qiuxy@bjmu.edu.cn

Professor Cui-Ying Li, Central Laboratory, Peking University School and Hospital of Stomatology, 22 South Zhongguancun Avenue, Haidian, Beijing 100081, P.R. China

E-mail: licuiying_67@163.com

*Contributed equally

Key words: salivary adenoid cystic carcinoma, cancer cell-derived immunoglobulin G, prognosis, epithelial-mesenchymal transition the expression of F-actin filaments. Taken together, these results showed that the high expression of cancer-IgG was strongly associated with metastasis, recurrence and invasion in SACC, suggesting that cancer-IgG expression could serve as a useful biomarker to predict the prognosis of the disease.

\section{Introduction}

Salivary adenoid cystic carcinoma (SACC) is a highly malignant neoplasm, which accounts for $\sim 10 \%$ of salivary gland malignancies (1-3). In patients with SACC, distant metastasis and perineural invasion are frequently observed, even during early stages of the disease (4). These features are associated with a poor prognosis and a 5-year survival rate of $<41.8 \%$ (5). Several proteins, including the epidermal growth factor receptor, transforming growth factor $\beta-1$ and the extracellular matrix (ECM) component fibronectin (6-8), have been shown to affect the aggressiveness of SACC. However, the precise mechanisms mediating the aggressive invasiveness of SACC remain unclear. Therefore, novel markers that predict prognosis in patients with SACC require identification and novel treatments must be developed.

According to classical immunology, immunoglobulin (Ig) molecules are only produced by differentiated B-lymphocytes and plasma cells (9). However, multiple studies have shown that numerous $\operatorname{Igs}$, including $\operatorname{Ig} \mathrm{A}, \operatorname{Ig} \mathrm{M}$ and $\operatorname{IgG}$, can also be produced and secreted by cells of other lineages, including neurons, germ cells and epithelial cells. Notably, non-B cell-derived Igs, particularly IgG, have been found to be overexpressed in a number of types of cancer, including those of the colon, breast, lung, stomach, liver, cervix, ovary, pancreas and prostate gland (10-15). Furthermore, cancer cell-derived IgG (cancer-IgG) has been shown to be involved in the progression and survival of cancer cells. Our previous study found that IgG was expressed in epithelial tumor cells in the oral cavity, including in SACC cells (16), using commercial human $\mathrm{IgG}$ antibodies. However, the role of cancer-IgG in the progression of SACC remains unclear. 
The present study aimed to elucidate the role of cancer-IgG in SACC by using the monoclonal antibody RP215, which specifically recognizes an unidentified glycosylated epitope of the heavy chain of cancer-IgG (RP215-bound IgG) (17), to investigate the association between cancer-IgG and the clinicopathological characteristics of patients with SACC. Additionally, the effects of cancer-IgG on the proliferation, migration and invasion of SACC cells were examined to provide important insights into the role of cancer-IgG in SACC.

\section{Materials and methods}

Patient samples. SACC tissues, including adjacent non-tumor salivary gland tissues, were collected from 96 patients aged between 32 and 83 years (mean age, 54 years) at the Peking University School of Stomatology (Beijing, China) between January 2004 and December 2009 (Table I). Tissue was fixed in formalin, embedded in paraffin and sectioned $(4-\mu \mathrm{m})$ for analysis. Data regarding pathological and clinical characteristics and outcomes were collected in a review of medical records. Ethical approval for this study was granted by the Peking University Health Service Trust Research Ethics Committee.

Cell culture. Human salivary adenoid cystic carcinoma SACC-83 cells (18) were obtained from Professor Shengling Li (Peking University School of Stomatology, Beijing, China) and cultured in RPMI-1640 medium (Gibco; Thermo Fisher Scientific, Inc., Waltham, MA, USA) supplemented with $10 \%$ fetal bovine serum (FBS; HyClone Laboratories, Logan, UT, USA) at $37^{\circ} \mathrm{C}$ with $5 \% \mathrm{CO}_{2}$.

Immunohistochemistry (IHC). The RP215 monoclonal antibody was a gift from Professor Gregory Lee (University of British Columbia, Vancouver, BC, Canada). Primary tumor tissues were sectioned into $4-\mu \mathrm{m}$ thick slices, deparaffinized and rehydrated using a graded ethanol series. Antigen retrieval was conducted by boiling the samples in Tris-EDTA buffer (pH 9.0) for $2 \mathrm{~min}$. All tissues were incubated with the RP215 primary antibody (1:200 dilution) at $4^{\circ} \mathrm{C}$ overnight or $37^{\circ} \mathrm{C}$ for $1 \mathrm{~h}$. Tissues were then washed thoroughly with PBS, incubated with horseradish peroxidase (HRP)-conjugated goat anti-mouse polyclonal antibodies (1:200, cat. no. ab97022; Abcam, Cambridge, UK) at $37^{\circ} \mathrm{C}$ for $20 \mathrm{~min}$. The immunocomplexes were detected using diaminobenzidine (Beijing Zhongshan Golden Bridge Biotechnology Co., Ltd., Beijing, China). The number of positive cells and the intensity of staining were determined in five randomly chosen fields at x200 magnification. The percentage of stained cells per field was graded as follows: 0 , Negative; $1,1-25 \%$ of cells; 2 , $26-50 \%$ of cells; and 3, 51-100\% of cells. The staining intensity was graded as follows: 0 , Absence of signal; 1, light brown for low-intensity signal; 2, brown for a moderate-intensity signal; and 3, dark brown for a high-intensity signal. The score for each field was obtained by multiplying the frequency grade and the intensity grade; the final score for each section was an average of all five fields. The scores for RP215 staining were described as negative (-) if the field scored $0-1$, low expression for a score of 2-3 (+), and high expression for scores of 4-6 and
7-9 (++ and +++, respectively). All evaluations were conducted using a Leica DM4000B/M microscope (Leica Microsystems, Inc., Buffalo Grove, IL, USA).

Western blot analysis. Western blot analysis was performed using standard procedures. Cells were lysed using RIPA buffer with protease inhibitor cocktail (Roche Diagnostics, Basel, Switzerland). Proteins (50 $\mu \mathrm{g})$ were transferred to nitrocellulose membranes following separation by $12.5 \%$ SDS-PAGE, and the membranes were then probed with the following antibodies overnight at $4^{\circ} \mathrm{C}$ : RP215 $(1 \mu \mathrm{g} / \mathrm{ml})$, anti-E-cadherin (cat. no. ab184633, dilution 1:1,000) and anti- $\beta$-actin (cat. no. ab8226, $1 \mu \mathrm{g} / \mathrm{ml}$ ) (both from Abcam). HRP-conjugated secondary antibodies (1:2,000, cat. no. ab97040; Abcam) were used to stain the membranes at room temperature for $1 \mathrm{~h}$. Immunocomplexes were then detected with the eECL Western Blot kit (cat. no. CW00495; Beijing ComWin Biotech Co., Ltd., Beijing, China).

RNA extraction, reverse transcription-polymerase chain reaction $(P C R)$. Total RNA was isolated from cells using TRIzol Reagent (Thermo Fisher Scientific, Inc.) and then reverse transcribed into cDNA using a SuperScript First-Strand Synthesis system (Thermo Fisher Scientific, Inc.). Quantitative PCR was conducted as previously described (6). The primers used for gene expression are listed in Table II.

Short interfering RNA (siRNA) synthesis and IgG-knockdown assay. siRNAs (Table III) targeting the constant region of the heavy chain in non-B cell-derived IgG were synthesized by Shanghai GenePharma Co., Ltd. (Shanghai, China). SACC-83 cells were seeded in 6-well culture plates at a density of $1 \times 10^{6}$ cells/well. After $24 \mathrm{~h}$, when the SACC-83 cells had grown to $50-70 \%$ confluence, the siRNAs and a non-targeting control (NC) were transfected into the SACC-83 cells using Lipofectamine 2000 (Thermo Fisher Scientific, Inc.) in RPMI-1640 without FBS for $6 \mathrm{~h}$. The medium was then replaced with complete medium without penicillin and streptomycin according to the manufacturer's instructions. The knockdown efficiency was verified by western blot analysis.

Colony formation and population doubling time (PDT) assays. The number of colony forming units (CFU) and the PDTs were assayed as described in our previous study (6). In brief, cells were seeded in 100-mm dishes, and aggregates of $>50$ cells were defined as a CFU after 14 days of incubation. For PDT assays, the cells were seeded in 96-well plates and counted daily.

Wound healing and invasion assays. As described in our previous study (6), IgG-knockdown and control cells were seeded into 6-well plates, grown to confluence and a scratch was made using a $300-400-\mu \mathrm{m}$ pipette tip. The average linear speed of movement of the wound edges was quantified over $24 \mathrm{~h}$. Cell invasion assays were performed using Transwell chambers coated with $20 \mu \mathrm{g}$ ECM gel (Sigma-Aldrich; Merck KGaA, Darmstadt, Germany). After 20 h, the membranes were stained with $1 \%$ crystal violet and cells on the upper surface of the membrane were removed using a cotton 
Table I. Association between cancer-IgG expression and clinicopathological parameters in patients with salivary adenoid cystic carcinoma.

\begin{tabular}{|c|c|c|c|c|}
\hline $\begin{array}{l}\text { Clinical } \\
\text { characteristics }\end{array}$ & $\begin{array}{l}\text { Cases } \\
\text { (n) }\end{array}$ & $\begin{array}{l}\text { Low cancer-IgG } \\
\text { expression }(n, \%)\end{array}$ & $\begin{array}{l}\text { High cancer-IgG } \\
\text { expression }(n, \%)\end{array}$ & $\chi^{2} \mathrm{P}$-value \\
\hline \multicolumn{5}{|l|}{ Age (years) } \\
\hline$<50$ & 44 & $23(52.3)$ & $21(47.7)$ & \multirow[t]{2}{*}{0.550} \\
\hline$\geq 50$ & 52 & $24(46.2)$ & $28(53.8)$ & \\
\hline \multicolumn{5}{|l|}{ Sex } \\
\hline Male & 36 & $16(44.4)$ & $20(55.6)$ & \multirow[t]{2}{*}{0.345} \\
\hline Female & 60 & $11(18.3)$ & $49(81.7)$ & \\
\hline \multicolumn{5}{|l|}{ Tumor size } \\
\hline$<30 \mathrm{~mm}$ & 41 & $24(58.5)$ & $17(41.5)$ & \multirow[t]{2}{*}{0.674} \\
\hline$\geq 30 \mathrm{~mm}$ & 55 & $35(63.6)$ & $20(36.4)$ & \\
\hline \multicolumn{5}{|l|}{ Histological subtype } \\
\hline Solid & 31 & $9(29.0)$ & $22(71.0)$ & \multirow[t]{2}{*}{0.767} \\
\hline Cribriform/tubular & 65 & $17(26.2)$ & $48(73.8)$ & \\
\hline \multicolumn{5}{|l|}{ Metastasis } \\
\hline Positive & 42 & $14(33.3)$ & $28(66.7)$ & \multirow[t]{2}{*}{$0.019^{\mathrm{a}}$} \\
\hline Negative & 54 & $31(57.4)$ & $23(42.6)$ & \\
\hline \multicolumn{5}{|l|}{ Nerve invasion } \\
\hline Yes & 35 & $11(31.4)$ & $24(68.6)$ & \multirow[t]{2}{*}{$0.009^{\mathrm{b}}$} \\
\hline No & 61 & $36(59.0)$ & $25(41.0)$ & \\
\hline \multicolumn{5}{|l|}{ Recurrence } \\
\hline Yes & 60 & $18(30.0)$ & $42(70.0)$ & \multirow[t]{2}{*}{$0.013^{\mathrm{a}}$} \\
\hline No & 36 & $20(55.6)$ & $16(44.4)$ & \\
\hline
\end{tabular}

${ }^{\mathrm{a}} \mathrm{P}<0.05 ;{ }^{\mathrm{b}} \mathrm{P}<0.01$. Cancer-IgG, cancer cell-derived immunoglobulin $\mathrm{G}$.

swab. Cells that had invaded through the membrane were then counted in at least 5 randomized fields under a light microscope. The results were obtained from at least three individual experiments.

Statistical analysis. Correlations between non-B cell-derived IgG expression and clinicopathological features, including age, sex, local invasion, recurrence and metastasis, were assessed using $\chi^{2}$ tests (for positive rates) and Mann-Whitney tests (for staining scores). Kaplan-Meier survival curves were used to estimate survival rates in patients with SACC. Comparisons of experimental data were performed to determine differences between knockdown and control SACC cells. Experiments were performed in triplicate. $\mathrm{P}<0.05$ was considered to indicate statistical significance. All analyses were performed using SPSS 19 software (IBM SPSS, Armonk, NY, USA).

\section{Results}

Non-B cell-derived IgG expression in SACC. IgG expression was investigated in SACC samples by immunohistochemical staining with RP215. Positive RP215 signals were primarily detected in the cytoplasm and plasma membrane of the cancer cells, whereas no signal was detected in mesenchymal cells or healthy salivary gland tissue. However, certain cells in the normal glandular ducts exhibited strong staining with RP215 (Fig. 1).

Next, whether $\operatorname{IgG}$ expression was correlated with various clinicopathological parameters in patients with SACC was investigated using IHC. Although there was no significant association between positivity of RP215 staining and sex, age (Table I), tumor size (data not shown), histological subtype, the level of RP215 staining was significantly associated with metastasis $(\mathrm{P}=0.019)$, recurrence $(\mathrm{P}=0.013)$ and nerve invasion $(\mathrm{P}=0.009)$ (Table I). Indeed, the rate of metastasis $(66.7 \%)$, recurrence $(70.0 \%)$ and nerve invasion $(68.6 \%)$ was higher in patients with high expression of non-B cell-derived $\mathrm{IgG}$ than in those with low expression of $\operatorname{IgG}(33.3,30$ and $31.4 \%$, respectively). The discrepancy between high and low RP215 staining in SACC tissues is shown in Fig. 2; strong positive staining was observed in cases with metastasis, recurrence and nerve invasion.

High cancer-IgG expression is associated with poor overall survival in patients with SACC. With the score for RP215 staining of cancer-IgG expression ( $\mathrm{T} / \mathrm{N}$ ) chosen as the cut-off point, the patients were divided into high- and low-expression groups. The patients with high cancer-IgG expression exhibited significantly shorter survival times than those with low cancer-IgG expression (Fig. 3). The results therefore showed that low cancer-IgG expression in SACC was associated with a poor prognosis. 
Table II. Primers used for quantitative polymerase chain reaction.

\begin{tabular}{llc}
\hline Primers & \multicolumn{1}{c}{ Sequence (5'-3') } & Gene ID \\
\hline E-cadherin & Forward: TGCCCCCAATACCCCAGCGT & NM_004360.3 \\
Vimentin & Foverse: TCCCTGTCCAGCTCAGCCCG & NM_003380.3 \\
MMP9 & Reverse: GGTCATCGTGATGCTGAGAAG & NM_004994.2 \\
MMP2 & Forward: GAGCCACGGCCTCCAACCAC & NM_001127891.2 \\
Slug & Foverse: GAGTCCAGCTTGCGGGGCAG & \\
& Reverse: CACCAGTGCCTGGGGCGAAG & NM_005985.3 \\
Twist & Forward: GAGCATTTGCAGACAGGTCA & NM_000474.3 \\
ZEB1/2 & Reverse: CCTCATGTTTGTGCAGGAGA & \\
& Forward: TCTCGGTCTGGAGGATGGAG & NM_001174094.1 \\
& Reverse: GTTATCCAGCTCCAGAGTCT & Forward: AGCAGTGAAAGAGAAGGGAATGC \\
& Reverse: GGTCCTCTTCAGGTGCCTCAG &
\end{tabular}

MMP, matrix metalloproteinase; ZEB1/2, zinc finger E-box binding homeobox 1/2.

Table III. siRNA sequences used for knockdown.

\begin{tabular}{lc}
\hline Name & Sequence $\left(5^{\prime}-3^{\prime}\right)$ \\
\hline siRNA NC & UUCUCCGAACGUGUCACGU \\
SiRNA1 & GGUGGACAAGACAGUUGAG \\
siRNA2 & AGUGCAAGGUCUCCAACAA \\
\hline
\end{tabular}

siRNA, short interfering RNA; NC, non-targeting control.

Proliferation and motility of cancer cells is suppressed by knockdown of non-B cell-derived IgG. Two siRNAs that target the constant region of the heavy chain of cancer-IgG were used to knockdown cancer-IgG expression in SACC-83 cells. Consistent with the analysis of clinicopathological characteristics, downregulation of cancer-IgG resulted in reduced cellular motility as evidenced by wound healing assays, in which the healing speed of the IgG-knockdown groups was significantly lower in SACC tissues than in control tissues (Fig. 4A). Moreover, cancer-IgG-knockdown decreased the invasion of SACC cells (Fig. 4B). Reduced IgG also suppressed colony formation (Fig. 4C) and increased PDT (Fig. 4D). The results of assays analyzing CFUs, PDT, speed and cell numbers after knockdown of IgG in SACC are shown in Table IV.

Knockdown of cancer-IgG suppresses the epithelial-mesenchymal transition (EMT) in SACC cells. To examine whether cancer-IgG was involved in the EMT, changes in the expression of EMT-associated molecules were evaluated. Knockdown of cancer-IgG significantly enhanced the expression of E-cadherin (Fig. 5A), which is negatively correlated with the EMT. By contrast, the expression levels of Twist, Slug and ZEB1/2, which are positively correlated with the EMT, were clearly decreased. In addition, IgG-knockdown caused the
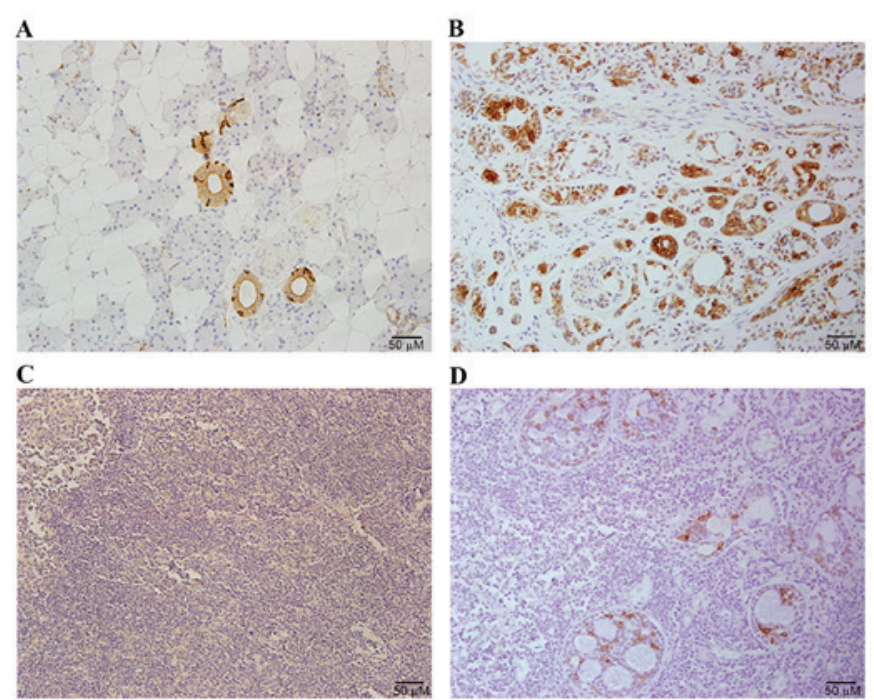

D

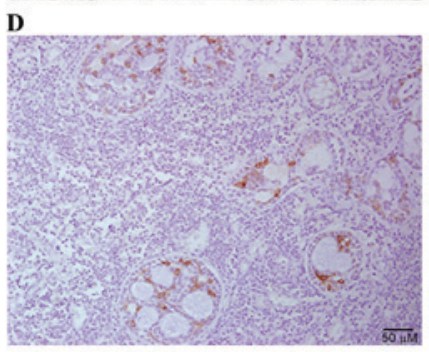

Figure 1. Cancer cell-derived immunoglobulin $\mathrm{G}$ was expressed in the SACC (A) Negative staining was observed in normal salivary gland tissues, whereas strong staining was observed in certain cells of the normal glandular duct. (B) Strong staining was observed as brown particles in the cytoplasm of SACC cells, particularly in the cytoplasmic membrane. No positive staining was observed in $(\mathrm{C})$ the lymph node, whereas positive staining was observed in (D) tumor cells that had invaded into the lymph node. SACC, salivary adenoid cystic carcinoma. Original magnification, 200; scale bar, $50 \mu \mathrm{m}$.

downregulation of matrix metalloproteinases 2 and 9 (MMP2 and MMP9), whose expression is associated with the invasion and metastasis of cancer cells (although the downregulation of MMP2 was not significant) (Fig. 5B). Furthermore, transfection with IgG siRNA altered the morphology of the SACC cells, resulting in a polygonal shape and a reduction in membrane ruffles, filopodia, lamellipodia and extensions when compared with control cells, in which F-actin tended to be arranged as filaments and cells exhibited a spindle shape (Fig. 5C). 
Table IV. CFU, PDT, cell speed and cell number following IgG-knockdown.

\begin{tabular}{|c|c|c|c|c|c|}
\hline Assays & Control $^{\mathrm{a}}$ & siRNA $1^{\mathrm{a}}$ & siRNA2 ${ }^{\mathrm{a}}$ & $\mathrm{P} 1^{\mathrm{b}}$ & $\mathrm{P} 2^{\mathrm{c}}$ \\
\hline CFUs (n) & $61.33 \pm 4.51$ & $32.33 \pm 2.52$ & $36.33 \pm 2.08$ & 0.015 & 0.021 \\
\hline PDT (h) & $49.67 \pm 3.79$ & $62.33 \pm 5.51$ & $63.67 \pm 6.66$ & 0.113 & 0.044 \\
\hline Speed $(\mu \mathrm{m} / \mathrm{h})$ & $18.58 \pm 0.52$ & $12.17 \pm 1.51$ & $12.58 \pm 0.80$ & 0.033 & 0.016 \\
\hline Cell no. & $107.33 \pm 5.87$ & $33.67 \pm 4.51$ & $36.33 \pm 1.53$ & 0.006 & 0.003 \\
\hline
\end{tabular}

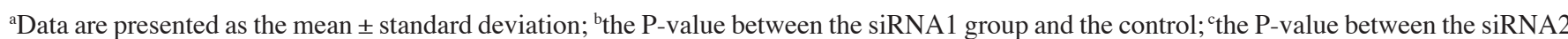
group and the control. siRNA, short interfering RNA; CFU, colony forming unit; PDT, population doubling time; IgG, immunoglobulin G.

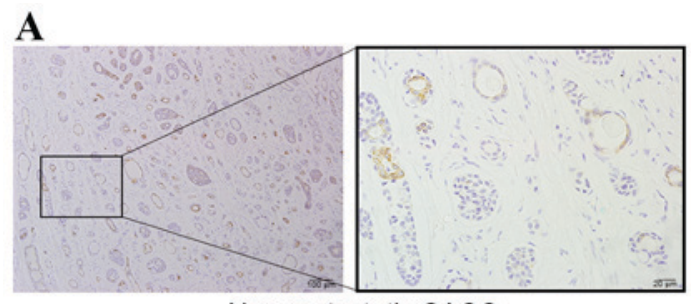

B

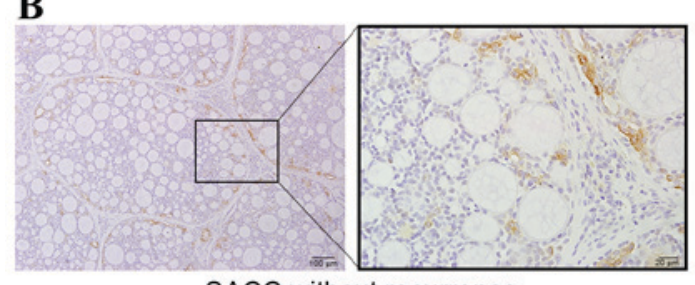

C

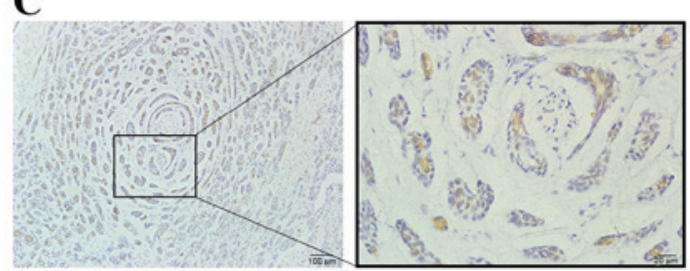

SACC without nerve invasion

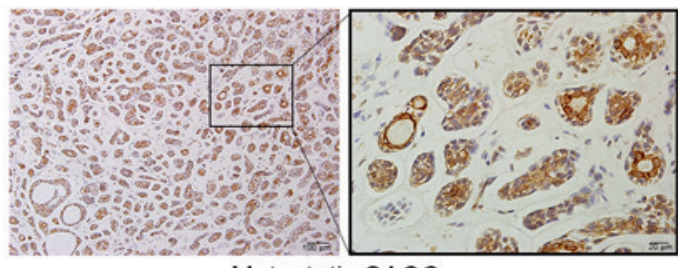

Metastatic SACC

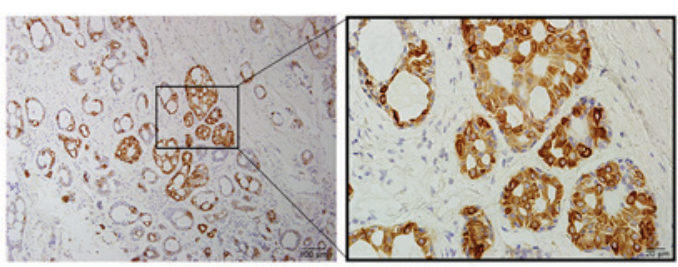

SACC with recurrence

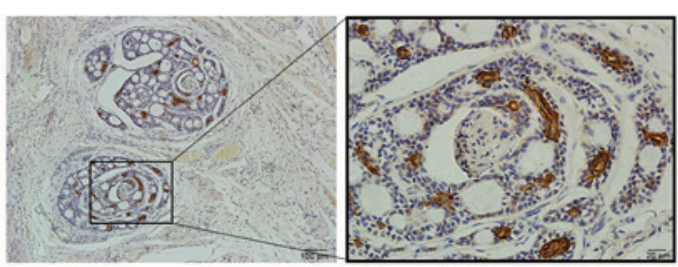

SACC with nerve invasion

Figure 2. Representative images of immunostaining in SACC tissues. (A) The expression of cancer-IgG in metastatic and non-metastatic tissues. (B) The expression of cancer-IgG in the tissues with or without recurrence. (C) The expression of cancer-IgG in the tissues with or without nerve invasion. Original magnification, x100 (left images) and x400 (right images). Cancer-IgG, cancer cell-derived immunoglobulin G; SACC, salivary adenoid cystic carcinoma.

\section{Discussion}

In the present study, IgG was found to be highly expressed in SACC tissues; this expression was found to be associated with metastasis, recurrence and nerve invasion in SACC. Additionally, IgG expression was involved in the proliferation, migration and invasion of SACC cell lines in vitro. These results provided important insights into the role of cancer-IgG and could facilitate the identification of biomarkers for SACC and the development of novel therapies for the treatment of the disease.

Studies have demonstrated that $\operatorname{IgG}$ is expressed in $\mathrm{B}$ cells and non-B cells. Additionally, IgG is overexpressed in various cancer types, including lung, colorectal and breast cancer $(11,13,19)$. In our previous study, the expression of IgG was detected in SACC tissues using commercial antibodies; however, recent studies have suggested that a monoclonal antibody, RP215, which mainly recognizes cancer-IgG, can

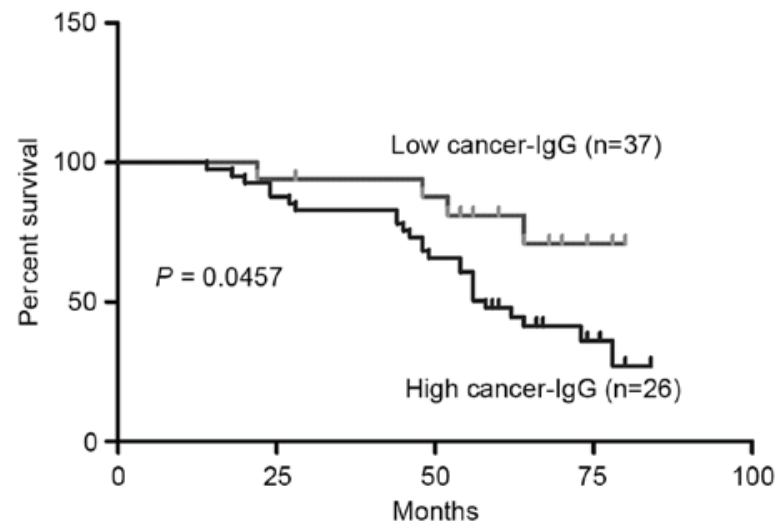

Figure 3. High cancer-IgG expression was significantly correlated with a poor prognosis in salivary adenoid cystic carcinoma. Kaplan-Meier curves with log-rank tests demonstrated that patients with high cancer-IgG expression exhibited significantly shorter survival times $(P=0.0457)$ compared with those in patients with low cancer-IgG expression. Cancer-IgG, cancer cell-derived immunoglobulin G. 
A

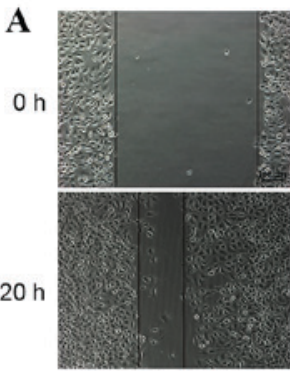

Control

B

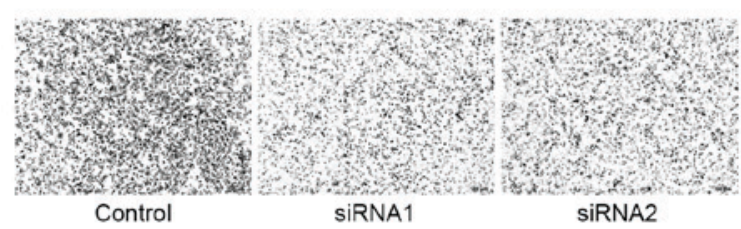

C

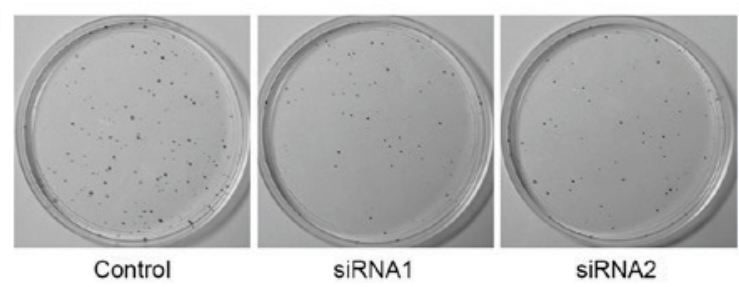

D

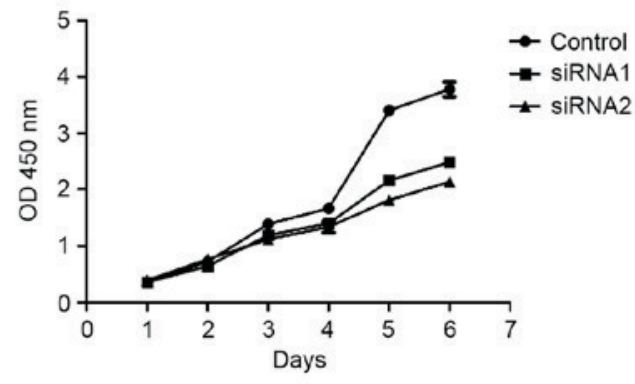

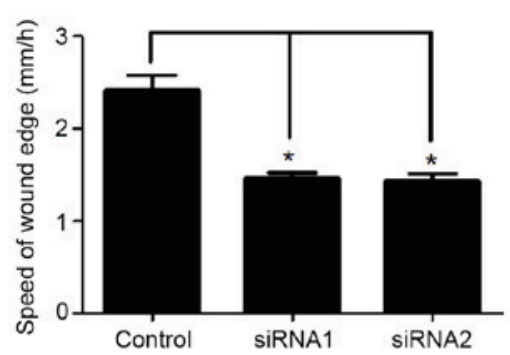
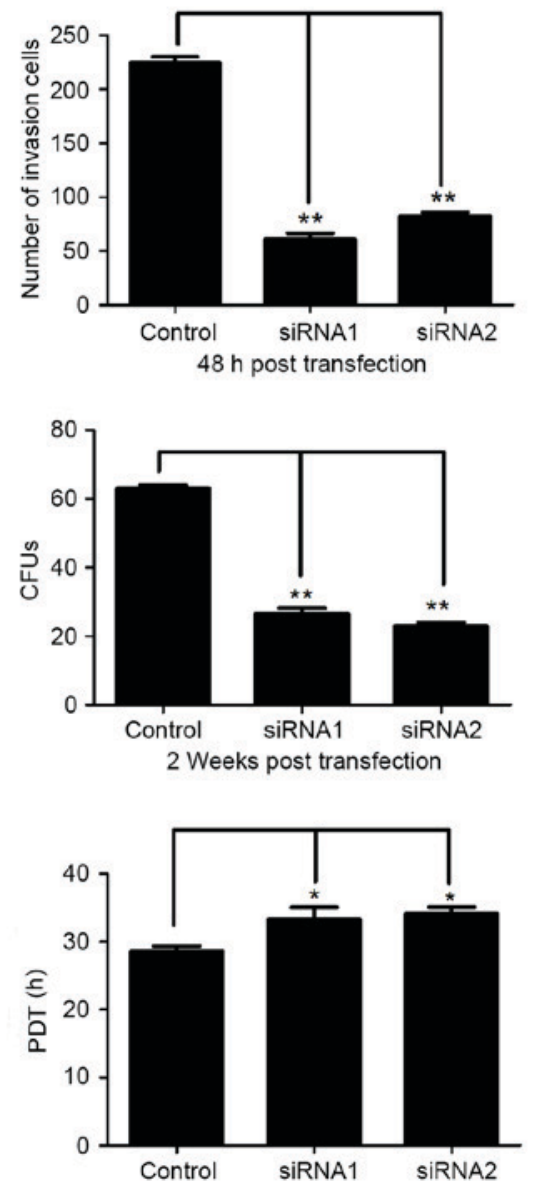

Figure 4. Proliferation and motility of cancer cells was suppressed by knockdown of cancer cell-derived IgG. (A) The speed of cell motility was reduced following knockdown of IgG expression in salivary adenoid cystic carcinoma as compared with that in the control group. Furthermore, the numbers of (B) invasive cells and $(\mathrm{C})$ colony forming units were decreased compared with those in control cells. (D) Cellular proliferation and PDT were also decreased. Data represent the mean \pm standard deviation of cells from at least three random fields under a microscope, and the experiment was repeated three times $\left({ }^{*} \mathrm{P}<0.05\right.$, $\left.{ }^{* *} \mathrm{P}<0.01\right)$. IgG, immunoglobulin G; SACC, salivary adenoid cystic carcinoma; CFU, colony forming unit; PDT, population doubling time; siRNA, short interfering RNA; OD, optical density.

be used to discriminate the origin of IgG between B cells and epithelial cancer cells $(20,21)$. In the present study, this antibody was used to detect cancer-IgG; it was found that IgG was significantly expressed in primary SACC tissues and an SACC cell line. In addition, adjacent normal salivary gland tissues, particularly gland tubular epithelial cells, also expressed cancer-IgG. However, further studies are required to determine the functions of cancer-IgG expressed by SACC.

In the present study, analysis of tissue from patients with SACC and corresponding clinicopathological characteristics showed that cases without metastasis, recurrence and nerve invasion exhibited a relatively low staining intensity compared with those cases in which metastasis, recurrence and nerve invasion occurred. Moreover, patients with high cancer-IgG expression (indicated by strong RP215 staining) exhibited a marked increase in the rates of metastasis, recurrence and nerve invasion compared with those having low cancer-IgG expression. High cancer-IgG expression was also associated with shorter survival times. Similar results have been reported in patients with lung adenoid carcinoma, further indicating that relatively high cancer-IgG expression may be associated with a poor prognosis (21). These results suggested that cancer-IgG was positively associated with the progression of SACC and may be a prognostic biomarker for the prediction of outcomes in patients with SACC. On the basis of the immunochemistry results, cancer- $\operatorname{IgG}$ may play 
A Cancer-IgG

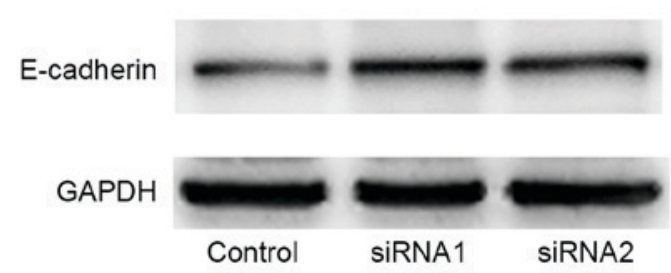

B

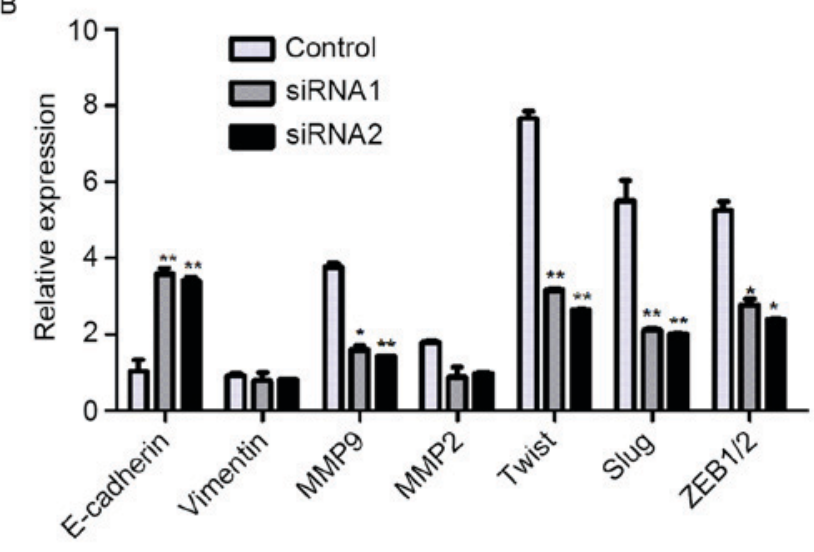

C

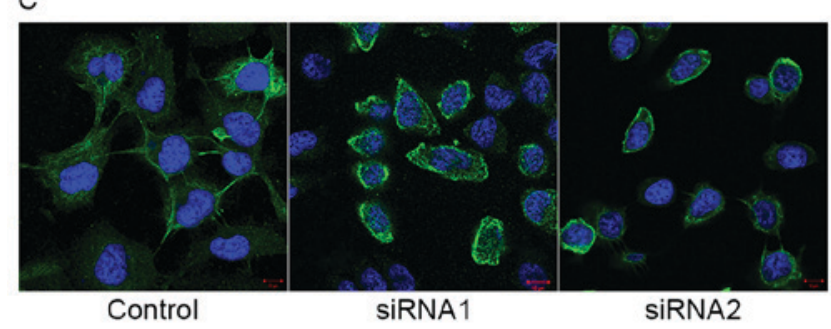

Figure 5. Knockdown of cancer-IgG suppressed the EMT in salivary adenoid cystic carcinoma cells. (A) The knockdown of IgG altered the expression of E-cadherin, as demonstrated by western blot analysis. (B) The mRNA levels of genes regulating the EMT were different to those of controls. (C) Immunofluorescence staining showed that F-actin filaments were concentrated in IgG-knockdown cells, whereas control cells exhibited an increased number of F-actin filaments and ruffles. Original magnification, $\mathrm{x} 400$; scale bar, $20 \mu \mathrm{m}$. Cancer-IgG, cancer cell-derived immunoglobulin $\mathrm{G}$; EMT, epithelial-mesenchymal transition; GAPDH, glyceraldehyde-3 phosphate dehydrogenase; siRNA, short interfering RNA; MMP, matrix metalloproteinase 9; ZEB1/2, zinc finger E-box binding homeobox $1 / 2$. ${ }^{*} \mathrm{P}<0.05,{ }^{* *} \mathrm{P}<0.01$.

an important role in the metastasis and invasion of SACC. However, further studies are required to confirm these initial findings.

To date, IgG secreted from cancer cells has been hypothesized to function primarily as an antibody. However, studies have also shown that cancer-IgG has growth factor-like activity and is involved in tumorigenesis, promoting the invasion and survival of cancer cells $(20,21)$. In the present study, it was found that SACC cell-derived IgG could promote cell proliferation in SACC-83 cells and that SACC cell-derived IgG significantly promoted cell invasion and migration in SACC-83 cells. Furthermore, cancer-IgG-knockdown suppressed the EMT, as evidenced by the increased expression levels of E-cadherin and decreased levels of Twist, Slug and ZEB1/2, as well as the reduced numbers of lamellipodia and disruption of F-actin filament arrangement. Moreover, MMP9 expression was reduced following cancer-IgG-knockdown, further supporting the fact that the invasive capacity of the tumor cells was impaired. It is possible that cancer-IgG may be associated with the invasion and migration of SACC through induction of the EMT.

In conclusion, the findings of the present study demonstrated that high expression of cancer-IgG was strongly correlated with metastasis, recurrence, nerve invasion and poor prognosis in patients with SACC. In addition, cancer-IgG also promoted cellular motility, aggressiveness and proliferation of SACC cells in vitro. These findings suggest that cancer-IgG can serve as a novel biomarker for predicting the prognosis of SACC and as a potential target for novel cancer therapies.

\section{Acknowledgements}

This study was supported by the National Nature Science Foundation of China (grant nos. 81072214, 81171006 and 81272237).

\section{References}

1. van der Wal JE, Becking AG, Snow GB and van der Waal I: Distant metastases of adenoid cystic carcinoma of the salivary glands and the value of diagnostic examinations during follow-up. Head Neck 24: 779-783, 2002.

2. Tian Z, Li L, Wang L, Hu Y and Li J: Salivary gland neoplasms in oral and maxillofacial regions: A 23-year retrospective study of 6982 cases in an eastern Chinese population. Int J Oral Maxillofac Surg 39: 235-242, 2010.

3. Wang YY, Chen WL, Huang ZQ, Yang ZH, Zhang B, Wang JG, Li HG and Li JS: Expression of the membrane-cytoskeletal linker Ezrin in salivary gland adenoid cystic carcinoma. Oral Surg Oral Med Oral Pathol Oral Radiol Endod 112: 96-1042, 2011.

4. Kokemueller H, Eckardt A, Brachvogel P and Hausamen JE: Adenoid cystic carcinoma of the head and neck - a 20 years experience. Int J Oral Maxillofac Surg 33: 25-31, 2004.

5. Liang LZ, Ma B, Liang YJ, Liu HC, Zheng GS, Zhang TH, Chu M, Xu PP, Su YX and Liao GQ: High expression of the autophagy gene Beclin-1 is associated with favorable prognosis for salivary gland adenoid cystic carcinoma. J Oral Pathol Med 41: 621-629, 2012.

6. Wang HC, Yang Y, Xu SY, Peng J, Jiang JH and Li CY: The CRISPR/Cas system inhibited the pro-oncogenic effects of alternatively spliced fibronectin extra domain A via editing the genome in salivary adenoid cystic carcinoma cells. Oral Dis 21: 608-618, 2015.

7. Ge MH, Ling ZQ, Tan Z, Chen C, Xu JJ and Yu JL: Expression of epidermal growth factor receptor in salivary adenoid cystic carcinoma and its role in cancer invasion. Zhonghua Zhong Liu Za Zhi 34: 278-280, 2012 (In Chinese).

8. Dong L, Wang YX, Li SL, Yu GY, Gan YH, Li D and Wang CY: TGF-beta1 promotes migration and invasion of salivary adenoid cystic carcinoma. J Dent Res 90: 804-809, 2011.

9. Arnold A, Cossman J, Bakhshi A, Jaffe ES, Waldmann TA and Korsmeyer SJ: Immunoglobulin-gene rearrangements as unique clonal markers in human lymphoid neoplasms. New Engl J Med 309: 1593-1599, 1983.

10. Qiu X, Zhu X, Zhang L, Mao Y, Zhang J, Hao P, Li G, Lv P, Li Z, Sun $X$, et al: Human epithelial cancers secrete immunoglobulin $\mathrm{g}$ with unidentified specificity to promote growth and survival of tumor cells. Cancer Res 63: 6488-6495, 2003.

11. Zheng S, Cao J and Geng L: Structure and expression of colorectal cancer related Immunoglobulin novel gene SNC73. Zhonghua Yi Xue Za Zhi 81: 485-488, 2001 (In Chinese).

12. Chen $\mathrm{Z}$ and $\mathrm{Gu} \mathrm{J}$ : Immunoglobulin $\mathrm{G}$ expression in carcinomas and cancer cell lines. FASEB J 21: 2931-2938, 2007. 
13. Jiang C, Huang T, Wang Y, Huang G, Wan X and Gu J: Immunoglobulin $\mathrm{G}$ expression in lung cancer and its effects on metastasis. PLoS One 9: e97359, 2014.

14. Liu Y, Chen Z, Niu N, Chang Q, Deng R, Korteweg C and Gu J: $\mathrm{IgG}$ gene expression and its possible significance in prostate cancers. Prostate 72: 690-701, 2012.

15. Kimoto Y: Expression of heavy-chain constant region of immunoglobulin and T-cell receptor gene transcripts in human non-hematopoietic tumor cell lines. Genes Chromosomes Cancer 22: 83-86, 1998.

16. Zhu X, Li C, Sun X, Mao Y, Li G, Liu X, Zhang Y and Qiu X Immunoglobulin mRNA and protein expression in human oral epithelial tumor cells. Appl Immunohistochem Mol Morphol 16 : 232-238, 2008.

17. Lee $\mathrm{G}$ and Ge B: Cancer cell expressions of immunoglobulin heavy chains with unique carbohydrate-associated biomarker. Cancer Biomark 5: 177-188, 2009.
18. Li SL: Establishment of a human cancer cell line from adenoid cystic carcinoma of the minor salivary gland. Zhonghua Kou Qiang Yi Xue Za Zhi 25: 29-31, 1990 (In Chinese).

19. Babbage G, Ottensmeier CH, Blaydes J, Stevenson FK and Sahota SS: Immunoglobulin heavy chain locus events and expression of activation-induced cytidine deaminase in epithelial breast cancer cell lines. Cancer Res 66: 3996-4000, 2006.

20. Liu Y, Liu D, Wang C, Liao Q, Huang J, Jiang D, Shao W, Yin CC, Zhang Y, Lee G and Qiu X: Binding of the monoclonal antibody RP215 to immunoglobulin $\mathrm{G}$ in metastatic lung adenocarcinomas is correlated with poor prognosis. Histopathology 67: 645-653, 2015.

21. Liao Q, Liu W, Liu Y, Wang F, Wang C, Zhang J, Chu M, Jiang D, Xiao L, Shao W, et al: Aberrant high expression of immunoglobulin $\mathrm{G}$ in epithelial stem/progenitor-like cells contributes to tumor initiation and metastasis. Oncotarget 6: 40081-40094, 2015. 